Val

T. Arndt

Bioscientia Institut für Medizinische Diagnostik $\mathrm{GmbH}$, Ingelheim, Deutschland

Synonym(e) Grammäquivalent; Äquivalent

\section{Englischer Begriff Val}

Definition Historische, heute obsolete Einheit zur Konzentrationsangabe von Ionen.

Beschreibung Die dem Äquivalentgewicht numerisch entsprechende Grammmenge heißt „1 Grammäquivalent“ oder auch kurz „1 Val“". Dabei ist das Äquivalentgewicht als Quotient aus Formelgewicht (Summe der relativen Atommassen 1t. Formel einer Substanz) dividiert durch die Wertigkeit (Ladung) definiert. Hierdurch sollte der Tatsache Rechnung getragen werden, dass mehrwertige Ionen, z. B. Sulfat-Ionen $\left(\mathrm{SO}_{4}{ }^{2-}\right)$, Phosphat-Ionen $\left(\mathrm{PO}_{4}{ }^{3-}\right)$ oder Kalzium-Ionen $\left(\mathrm{Ca}^{2+}\right)$ eine 2- bzw. 3-mal stärkere ,wirksame“ Konzentration in einer Lösung entfalten als eine identische Einwaage einwertiger Ionen wie z. B. $\mathrm{Cl}^{-}, \mathrm{Na}^{+}$oder $\mathrm{K}^{+}$.

Lösungen, die je Liter 1 Val einer Substanz enthalten, werden „1-normale Lösungen“ genannt. In einer 1-normalen Sulfatlösung sind demnach 96,006:2 $=48,033 \mathrm{~g} \mathrm{SO}_{4}{ }^{2-}$, in einer 1-normalen Phosphatlösung sind 94,978:3 = 31,656 g $\mathrm{PO}_{4}{ }^{3-}$ gelöst.

Ältere Publikationen z. B. zu Normalbereichen (heute Referenzbereichen) von Ionen in Blut und Urin geben diese häufig mit der Einheit $\mathrm{mVal} / \mathrm{L}$ (engl.: $\mathrm{mEq} / \mathrm{L}$ ) an. Nach o. g. Ausführungen sind diese Angaben mit der Wertigkeit der betrachteten Ionen zu dividieren, vorausgesetzt, die Wertigkeit ist eindeutig definiert (wie z. B. für Kalzium- und $\mathrm{SO}_{4}{ }^{2-}$-Ionen) und es liegen nicht unterschiedliche Oxidationsstufen parallel vor (z. B. Phosphat, das in der Form $\mathrm{H}_{2} \mathrm{PO}_{4}^{-}, \mathrm{HPO}_{4}{ }^{2-}$ und $\mathrm{PO}_{4}{ }^{3-}$ vorliegt). Für einwertige Ionen wie z. B. $\mathrm{Cl}^{-}, \mathrm{Na}^{+}$oder $\mathrm{K}^{+}$entspricht der Zahlenwert der Konzentrationsangabe in $\mathrm{mVal} / \mathrm{L}$ jenem der Konzentrationsangabe in $\mathrm{mmol} / \mathrm{L}$ (Division durch „1").

\section{Literatur}

Wiberg N (1995) Holleman-Wiberg Lehrbuch der Anorganischen Chemie, 101. Aufl. Walter de Gruyter, Berlin/New York, S 163-164 